\title{
Difficult diagnosis of a neurogenic thoracic outlet syndrome and review of the current literature
}

\author{
João Maia Rosa*, Arnaldo Sousa, Hélder Fonte, Eva Pereira, Rita Sapage, Miguel Trigueiros
}

Orthopaedics Department, Centro Hospitalar e Universitário do Porto, Porto, Portugal

Received: 24 May 2020

Revised: 05 July 2020

Accepted: 13 July 2020

\author{
*Correspondence: \\ Dr. João Maia Rosa, \\ E-mail: jnmrosa@gmail@gmail.com
}

Copyright: (c) the author(s), publisher and licensee Medip Academy. This is an open-access article distributed under the terms of the Creative Commons Attribution Non-Commercial License, which permits unrestricted non-commercial use, distribution, and reproduction in any medium, provided the original work is properly cited.

\begin{abstract}
Thoracic outlet syndrome (TOS) is an uncommon disorder, without a clear clinical presentation or a fully accepted definition. The patients usually present a set of symptoms arising from compression of the brachial plexus or subclavian vessels during their passage through the thorax, between the base of the cervical region and the axilla. The authors present a case of a 20 year old woman with sporadic paresthesia in the territory of C7-T1 nerve root. At the physical examination she had a positive Wright's test. The imaging studies revealed the presence of a right cervical rib. A partial rib resection was performed with exploration of the braquial plexus and at the current follow up; 24 months after the surgery, the patient is asymptomatic. Our objective is to present a case of TOS and to highlight the clinical-management and treatment challenges of this pathology.
\end{abstract}

Keywords: Thoracic outlet syndrome, Cervical rib, Wright's test, Braquial plexus compression, Lower trunk

\section{INTRODUCTION}

Thoracic outlet syndrome (TOS) is an uncommon disorder with some controversies concerning its causes, diagnosis, and treatment despite years of intense study of hundreds of patients. ${ }^{1}$ Thoracic outlet syndrome is characterized by the presence of a compression of the structures at thoracic outlet such as subclavian artery, subclavian vein and brachial plexus. The main causes are the presence of a cervical rib, trauma, scalene muscle hypertrophy and fibrous band. ${ }^{2}$ Cervical rib is a congenital anomaly which is originated from enlargement of the transverse process of C7. This anomaly occurs $1 \%$ of the population but induces the symptoms about $5 \% .^{3}$ The symptoms may vary according to the compressed structure such as paleness, swelling, edema, numbness and pain. ${ }^{1}$ A normal electromyogram does not excludes its presence, as reported in our clinical case. ${ }^{4}$ As a result, the diagnosis of this syndrome is very defying.

\section{CASE REPORT}

A 20-year-old woman was referred to our hospital due to diffuse pain in the shoulder and right cervico-scapular region with 2 years of evolution. She had no history of trauma or prior surgery.

Initially attended to the shoulder unit consultation where a shoulder ultrasound was requested, which revealed no alterations and a cervical $\mathrm{Rx}$ which revealed the presence of an accessory cervical rib on the right side (Figure 1-3).

In a second consultation, when specifically questioned, she reported sporadic paresthesia in the territory of C7-T1 nerve root. At the physical examination, it was possible to reproduce these complaints during the abduction and external rotation of the right arm (Wright's test). An electromyogram was performed that revealed no evidence 
of brachial plexus injury or neuropathy in the upper limb or motor cervical radiculopathy.

The CT revealed the presence of a right cervical rib, which had immediate relation with the trunk inferior to the brachial plexus, but not to the subclavian artery (Figure 4 and 5).

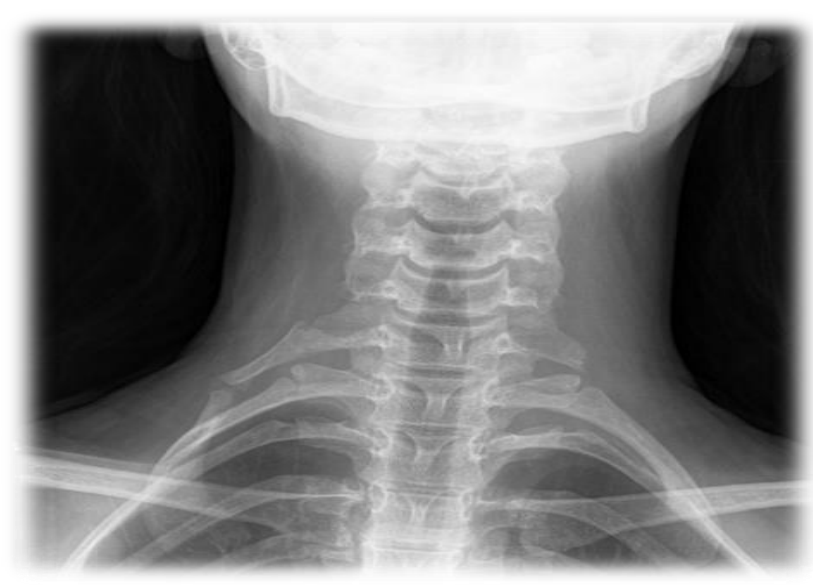

Figure 1: Cervical Rx AP view.

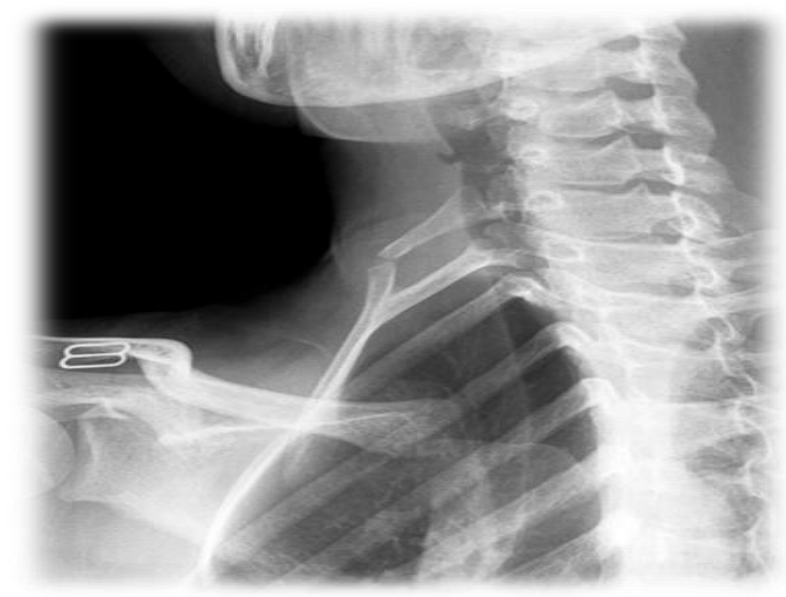

Figure 2: Cervical Rx oblique view.

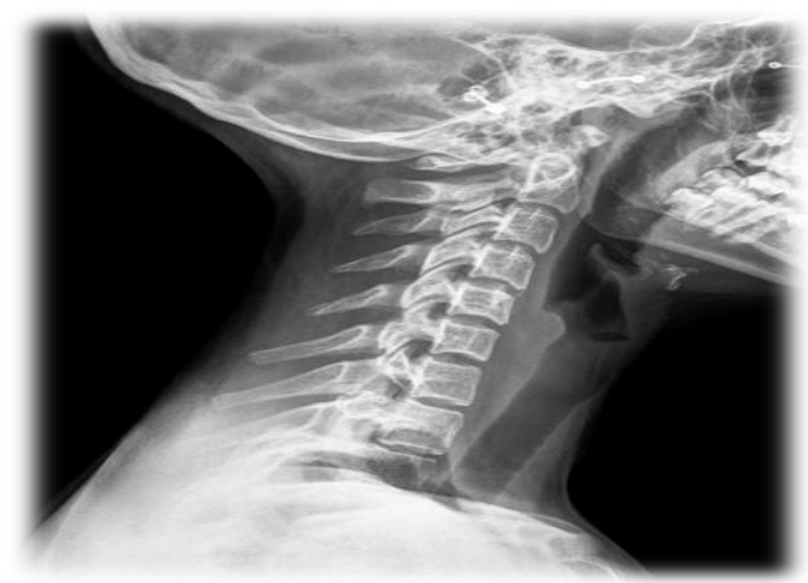

Figure 3: Cervical $\mathrm{Rx}$ lateral view.

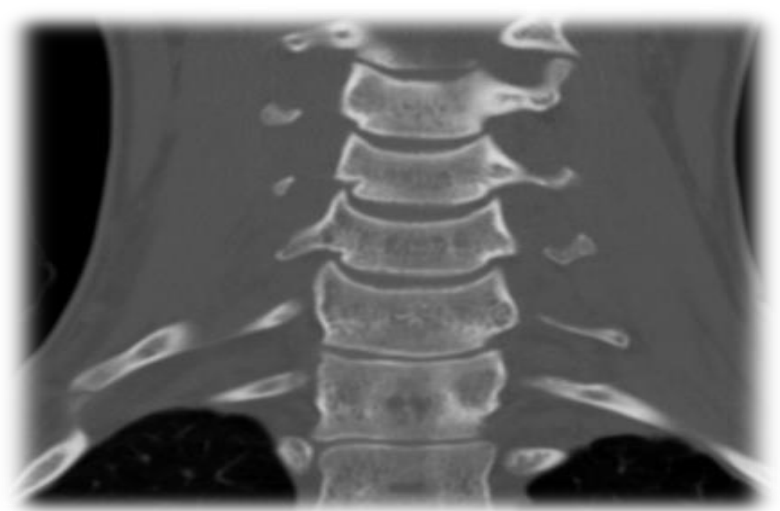

Figure 4: CT scan AP view.

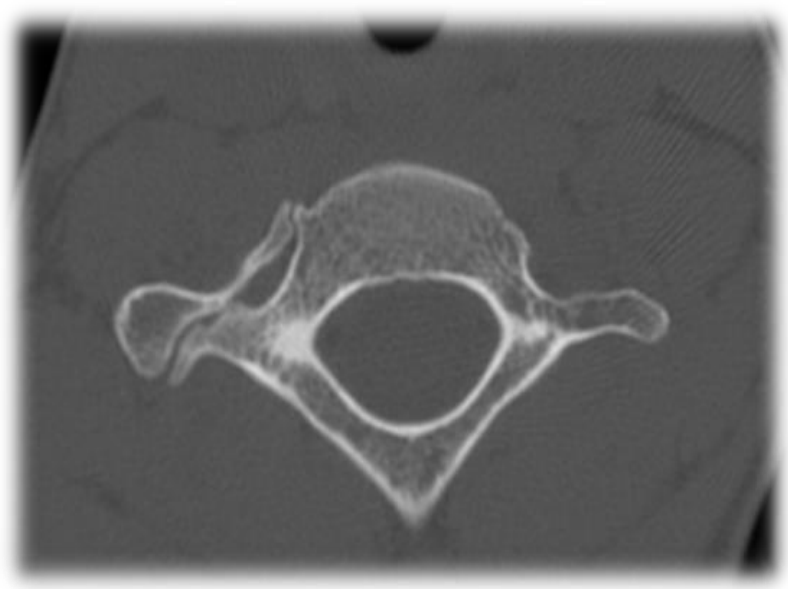

Figure 5: CT scan axial view (C7).

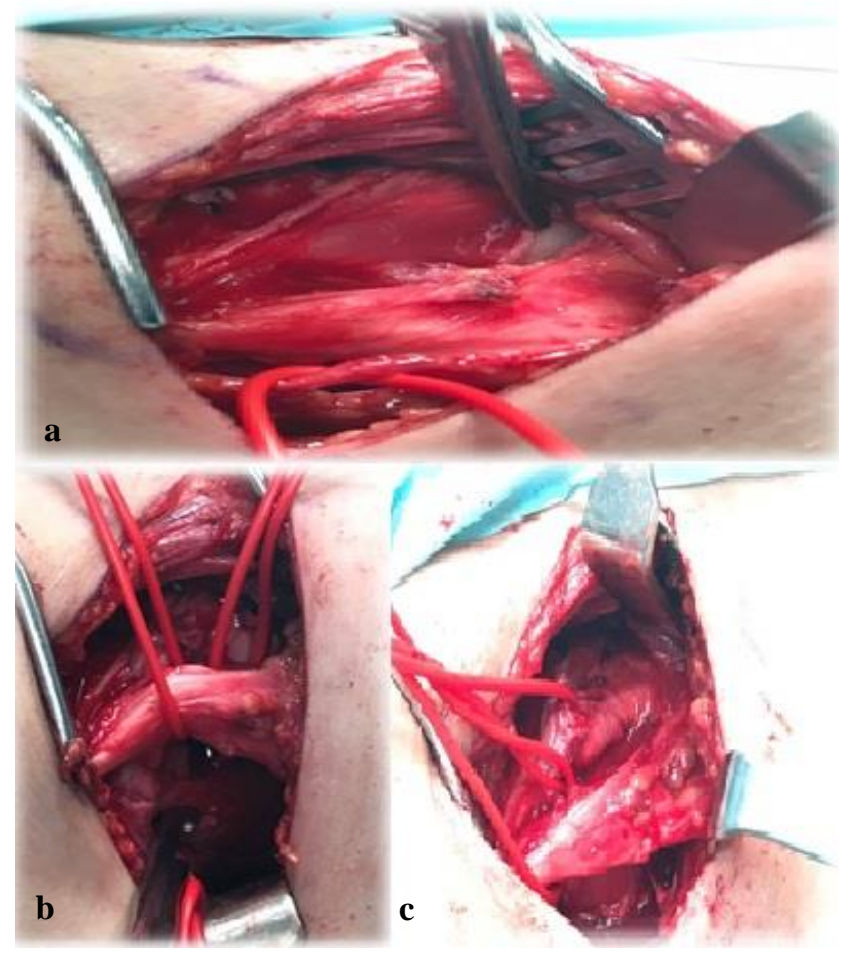

Figure 6: Intra-operative photos. 
Nearly 2 years after the first consultation, motivated by diffuse pain in the shoulder and right cervical-scapular region and a study directed at the shoulder and cervical radiculopathy with no etiology found and with no improvements with the conservative treatment, the patient was then submitted to surgical decompression of the lower brachial plexus with partial resection of the cervical rib using a right supraclavicular approach (Figure $6 \mathrm{a}-\mathrm{c}$ ).

About 24 months after surgery, the patient presented a complete resolution of pain and paresthesia in the C7-T1 territory.

\section{DISCUSSION}

Most individuals with nTOS are young female. According to the literature, there are several possible etiologies for TOS, both congenital and acquired. The most common are scalene hypertrophy, congenital cervical ribs, first rib and clavicle anomalies or fibrotic bands.

Even so, the presence of a cervical rib is not synonymous with TOS. ${ }^{5}$ The prevalence of cervical ribs in the population is $0.5-2 \%$ and the prevalence of nTOS is 1 per million, so the ratio of cervical ribs to nTOS is 500020,000 to 1 . Thus, the presence of a cervical rib in an individual with nonspecific symptoms of the upper extremities is probably only an incidental finding. ${ }^{6}$

The vast majority of cases of TOS are delayed in diagnosis due to confounding factors such as associated comorbidities, like psychiatric disorders or those related to the shoulder joint and even so they may be associated with a normal electromyogram that is not sensitive at an early stage. $^{4}$

This case report highlights the importance of this syndrome in the differential diagnosis of cervical pain or radiculopathy, as well as the good results of its surgical treatment, with success rates above $90 \%$ being described. ${ }^{7}$

\section{CONCLUSION}

Many patients with TOS benefit from surgical treatment to resolve their complaints. However, the difficulties in obtaining a clear diagnosis, as well as the socio-economic characteristics and associated diseases of these patients can often delay the definitive treatment and thereby compromise the final clinical result. An adequate operative procedure performed by an experienced team is also crucial to success. ${ }^{8}$

\section{Funding: No funding sources \\ Conflict of interest: None declared \\ Ethical approval: Not required}

\section{REFERENCES}

1. Oates SD, Daley RA. Thoracic Outlet Syndrome. Hand Clinics. 1996;12:705-18.

2. Luoma A, Nelems B. Thoracic Outlet Syndrome: Thoracic Surgery Perspective. Neurosurg Clin North Am. 1991;2:187-226.

3. Sirasaporn P. Neurogenic Thoracic Outlet Syndrome: Causing by Cervical Rib. J Med Cases. 2014;5(2):120-22.

4. Cruz-Martínez A, Arpa J. Electrophysiological Assessment in Neurogenic Thoracic Outlet Syndrome. Electromyogr Clin Neurophysiol. 2001;41:253-6.

5. Ferrante MA, Ferrante ND. The Thoracic Outlet Syndromes: Part 1: Overview of the Thoracic Outlet Syndromes and Review of True Neurogenic Thoracic Outlet Syndrome. Muscle Nerve. 2017;55:782-93.

6. Henry BM, Vikse J, Sanna B, Taterra D, Gomulska M, Pekala PA et al. Cervical Rib Prevalence and its Association with Thoracic Outlet Syndrome: A Meta-Analysis of 141 Studies with Surgical Considerations. World Neurosurgery. 2018;110:96578.

7. Peek J, Vos CG, Ünlü Ç, Schreve MA, van de Mortel RHW, de Vries JPM. Long-Term Functional Outcome of Surgical Treatment for Thoracic Outlet Syndrome. Diagnostics (Basel, Switzerland). 2018;8:7.

8. Seifert S, Sebesta P, Klenske M, Esche M. Thoracic Outlet Syndrome. Zbl Chir. 2017;142:104-12.

Cite this article as: Rosa JM, Sousa A, Fonte H, Pereira E, Sapage R, Trigueiros M. Difficult diagnosis of a neurogenic thoracic outlet syndrome and review of the current literature. Int J Res Orthop 2020;6:1087-9. 